\title{
Modeling the egg packing station planning problem
}

\author{
Reginald Dewil $^{1}$, Johan Philips ${ }^{1}$ Jan Jaap Kempenaar ${ }^{2}$, and Dirk Cattrysse ${ }^{1}$ \\ 1 KU Leuven, Leuven, Belgium, \\ reginald.dewil@kuleuven.be, \\ 2 MOBA B.V., Stationsweg 117, 3771 VE, Barneveld, Netherlands, \\ WWW home page: http://www.moba.net/page/en
}

\begin{abstract}
This paper presents the planning problem encountered by egg packing station managers. In an egg packing station, pallets containing unsorted eggs arrive daily from chicken farms and need to be packed into order-specific boxes on packing lanes. The grader automatically identifies damaged eggs and grades the remaining eggs in several weight classes. The number of packing lanes is station specific but can reach up to 32 . The orders are destined for retailers and they specify the number of required eggs and a set of allowed grades. The challenge is to assign these orders to the packing lanes such that the incoming supply (distribution of grades) is completely covered by active orders on the packing lanes. This paper positions the egg packing problem in the optimization literature and proposes a model that efficiently covers this planning problem. Furthermore, we point out several future research directions such as additional practical work floor constraints and algorithmic challenges.
\end{abstract}

Keywords: order scheduling, egg grading, problem modeling

\section{Introduction}

Eggs are a major European export product. The European Commission forecasted an average monthly production of 7900 million eggs in the European Union in 2018 and with prices hovering around $€ 120$ per $100 \mathrm{~kg}$ for A-grade eggs, it is an important multi-billion euro industry[1]. In the European Union, eggs are typically produced in laying farms, palletized and sent to a packing station where the eggs are graded by an egg grader. Egg graders are large egg sorting machines that grade a continuous supply of incoming eggs into different weight classes, hereafter referred to as grades, and remove damaged and otherwise unsuitable eggs (dirty, rotten, bloody). The eggs are subsequently allocated to packing lanes where they are packed in carton boxes destined for retailers. Figure 1 shows an Omnia PX egg grader of machine builder MOBA with 16 packing lanes and 1 end lane.

The weight distribution of the incoming supply of eggs can be approximated by a normal distribution. Since the average weight and standard deviation changes very gradually as the chickens age, the weight distribution can be 


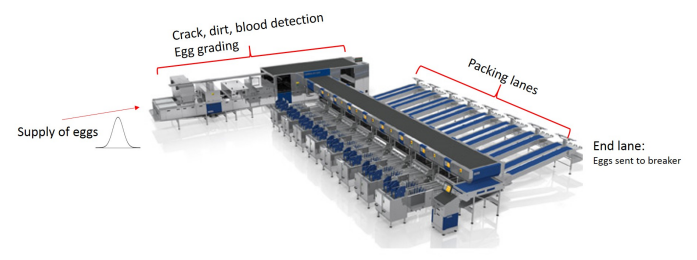

Fig. 1. An Omnia PX egg grader (MOBA B.V.)[2]

reliably estimated per egg laying farm based on the weight distribution of the egg production of the previous day. In the packing station, pallets coming from different farms are available at the start of the packing shift, each with their own weight distribution. Put otherwise, with every pallet change, the weight distribution of incoming eggs might change.

The orders specify the number of eggs required and the allowed grades. The number of eggs required for a given order can vary to a great extent: in the range of 200 eggs up to 160 thousand eggs. The allowed grades can be a single specific grade, such as extra large $(X L)$ eggs or be composed of multiple grades, such as: medium, large, and extra large $(M, L, X L)$. A single order can be fullfilled on multiple packing lanes simultaneously. Since the boxes destined for the same order need to be grouped to be palletized, it is preferable that these so-called sub orders are assigned to adjacent lanes in order to minimize any additional handling. However, this is not a hard constraint.

Figure 2 represents the planning problems in its entirety. The goal is to create sub order - lane allocation such that as many available orders are completely fullfilled given the available egg supply and profit is maximized. This implies minimizing the sequence-dependent setup times and minimizing the number of eggs sent to the end lane. Eggs sent to the end lane are destined for a breaker machine that separates yolk from white which are sold separately at much lower prices than an intact packaged egg. Eggs will be sent to the end lane if there is insufficient packing capacity available to process the egg's grade, or put otherwise, there are insufficient active orders that accept the egg's grade.

Given sub order-lane allocations, the grader will assign individual eggs to active orders that are allowed to pack that egg's grade using a heuristic that tries to assign the eggs equally to the packing lanes. Although some uncertainty remains on how long it will take until a similar grade egg will be graded, the small lane buffers are properly dimensioned to cover any short-term deviation from the normal distribution assumption. Therefore, egg flows resulting from a normally distributed supply can be safely used to determine the number of eggs being allocated to a specific lane. More specifically, equations 1 and 2 approximate the allocation heuristic used by the grader. First, an initial egg flow $f_{g, o}^{\text {initial }}$ per grade $g$ to each sub order $o$ is determined using equation 1 . Let $s_{g}$ be the egg supply per time unit of grade $g$ and $O_{g}$ be the set of active sub orders that have 


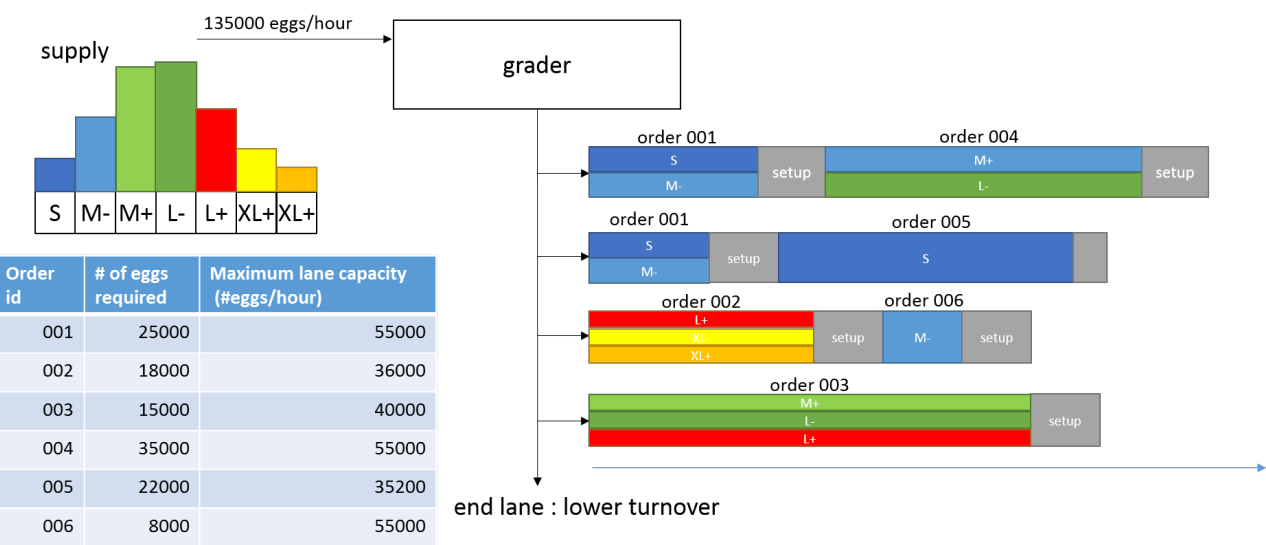

Fig. 2. The planning problem

grade $g$ as an allowed grade, then the initial egg flow for sub order $o$ of grade $g$ is:

$$
f_{g, o}^{\text {initial }}=s_{g} \frac{\frac{1}{\sum_{g^{\prime} \in \text { o.grades }} s_{g^{\prime}}}}{\sum_{r \in O_{g}} \frac{1}{\sum_{g^{\prime \prime} \in \text { r.grades }} s_{g^{\prime \prime}}}}
$$

This initial egg flow is subsequently adjusted if one of these flows would exceed the packing capacity of the lane using equation 2 .

$$
f_{g, o}=f_{g, o}^{\text {initial }} \frac{\text { min(o.lane.capacity } \left., \sum_{g \in \text { o.grades }} f_{g, o}^{\text {initial }}\right)}{\sum_{g \in \text { o.grades }} f_{g, o}^{\text {initial }}}
$$

Consequently, the eggs being sent to the end lane for each grade $g$ is the difference between the sum of the egg flows to each sub order and the total supply of that grade (equation 3).

$$
f_{g, \text { endlane }}=s_{g}-\sum_{o \in O_{g}} f_{g, o}
$$

We can conclude from these equations that if anything changes in the active lanes, a breakdown, an end or a start of a sub order on a lane, the egg flows to multiple lanes are affected since the number of active orders of a given grade changes. This has a major impact on the computational complexity of the scheduling problem since the packing lanes cannot be considered as independent machines. 


\section{Solution representation and evaluation}

In many basic production scheduling problems or routing problems, in order to result in a Gantt chart as depicted in figure 2, a simple two dimensional array of orders suffices. The rows represent the different machines, vehicles, or in this case, the lanes and the elements in the rows represent the sequence of jobs to be executed or stops to be visited. Each row is independent and the objective function value of a complete solution can be determined by adding the objective function values of the individual machine schedules or vehicle routes. This observation lies at the basis of the many successful local search or metaheuristic optimization approaches since a small perturbation can be efficiently evaluated within the route or machine itself and not bother with looping over the complete solution, and thus millions of new solutions can be evaluated in short computation times.

Since in the packing problem, the lane schedules are not independent and the egg flows directly impact the completion times of orders, it is not trivial to identify a local perturbation that can be evaluated in constant time. Whereas in typical scheduling problems, an order start time directly fixes the order end time, in this problem, the end time is dependent on the egg flows. As depicted in figure 3, these egg flows change at every event, e.g. start pallet, end pallet, start sub order, end sub order, start setup, end setup.
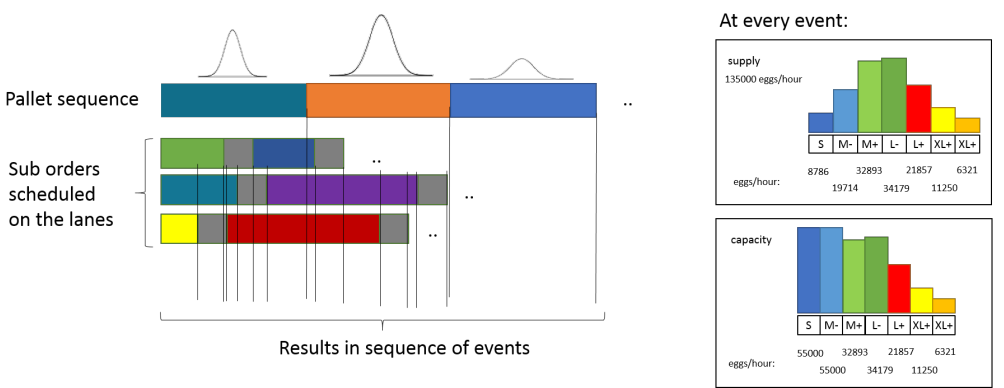

Fig. 3. A sequence of orders and pallets results in a sequence of events each with its own egg flow.

A single solution can then be evaluated by looping once over the solution and iteratively identifying the next event to occur based on the current egg flow and determining the new egg flow and the number of eggs processed of the pallet and to the sub orders at this event. This is an $\mathrm{O}(n+m)$ operation with $n$ being the number of sub orders and $m$ being the number of pallets. Given the efficacy of $\mathrm{O}\left(n^{2}\right)$ local move operators such as remove-reinsert and 2opt in similar routing or scheduling problems and the fact that $\mathrm{O}\left(n^{3}\right)$ local move operators are computationally acceptable for the problem sizes of daily instances 
( $\leq 100$ orders, $\leq 100$ pallets, $\leq 32$ lanes) leads us to conclude that using a classcial $\mathrm{O}\left(n^{2}\right)$ operator with the event-based $\mathrm{O}(n)$ evaluation will most likely lead to an effective optimization approach utilizing $\mathrm{O}\left(n^{3}\right)$ local move operators.

A construction heuristic was developed that greedily schedules orders on lanes as they become available. Its greedy criterion is to minimize the eggs being sent to the grader in the resulting egg flow. Ties are broken by selecting the order with the fewest eggs remaining to be fullfilled. In order to generate multiple initial solutions, the construction heuristic was implemented in a Greedy Randomized Adaptive Search Procedure (GRASP). The GRASP heuristic was run on a real-life benchmark from one of the more efficient egg packing stations in the Netherlands. Comparing to a schedule generated by a manual planner, the GRASP heuristic already improves the daily profit by $6.5 \%$.

In order to determine an upper bound on the achievable profit, the complete problem can also be modeled as a Transportation Problem: the sources are all the incoming eggs and the sinks are the complete set of orders. A source is created for each grade. Assuming a perfect utilization of the lanes and disregarding setups, an upper bound on the profit can be determined. The original schedule and the GRASP schedule showcase gaps of $13 \%$ and $7 \%$ respectively with this upper bound. This is a major motivation to continue the research not only in adding additional constraints and features but also in searching for better optimization approaches.

\subsection{Egg lane allocation}

The allocation equations imply that the more grades that are permitted by an active order, the less eggs will be assigned to that lane from a specific allowed grade. Furthermore, it implies that, for a specific grade, eggs will be sent to all lanes that are permitted to process this grade. This can actually lead to unwanted effects as can easily be seen through the following example. Consider two orders, each requiring 100 eggs. The first order can be fullfilled by Small or Medium eggs and the second order can only be fullfilled by Small eggs. Suppose that both orders are scheduled on separate lanes with infinite processing capacity. Furthermore, suppose that a supply of 100 Small and 100 Medium eggs is available. It follows that both orders can easily be fullfilled to completion. Assume that the grader speed is 100 eggs per second (eps). Therefore, there will be a supply of 50 eps of Medium eggs and 50 eps of Small eggs. However, the above defined egg allocation equations result in the following egg flows per second: $f_{S, 1}=16.67, f_{M, 1}=50, f_{S, 2}=33.33$. This means that there is an egg flow of 66.67 eggs per second to the first order which will be fullfilled after 1.5 seconds after which a supply of 25 Medium eggs remains which is not allowed to be allocated to the second order.

This simple example illustrates a problem that effectively occurs in practice. However, this problem is, on the one hand, exacerbated by lane capactity constraints which result in eggs being sent to the end lane and, on the other hand, alleviated by reallocating eggs to downstream lanes if no order-specific text is printed on them. 
From an Operations Research perspective, this allocation problem is clearly a Transportation Problem and initial experiments suggest that replacing the machine's heuristic by an optimal algorithm that solves the Tranportation Problem can improve daily profitability by an additional $2 \%$.

\section{Discussion and outlook}

The egg planning problem as presented above is a basic version of the planning problem as it occurs in practice. However, the results we have obtained using a very simple GRASP heuristic is sufficiently promising to continue this line of research. Furthermore, additional improvement potential is identified in improving the egg allocation heuristic and there remains improvement potential by implementing a local search phase in the optimization process.

In order to design a schedule that will be accepted by practicioners on the shop floor, many additional constraints need to be considered and the approach presented above is just a first step. In particular, the above approach does not place a limit on the number of setups that can be executed in parallel but in reality this is limited by the number of people executing the setups.

The packing lanes are subject to frequent short breakdowns which have a major impact on the egg flows. Consequently, the schedules should be constructed such that sufficient capacity for every grade is available at all times to cover one or multiple simultaneous breakdowns.

Sometimes, a single worker operates two lanes simultaneously or multiple packing lanes are linked together downstream by a packing robot. In both cases, the lane capacities are limited by the downstream robot or worker capacity. However, this capacity is shared across the lanes and introduces an extra degree of complexity in the egg allocation and consequently the order scheduling problem.

In the current version, we have assumed that the orders and pallets were given beforehand. However, this problem occurs daily and some orders can be postponed and, similarly, some egg supplies can be processed a day or two later. Hence, we should actually consider a multi-day planning problem.

The end goal is to develop an advanced planning module that can schedule the daily operations and reschedule quickly when unforseen events occur. Furthermore, such a module can be used to determine the optimal machine configuration for new packing stations supporting the commercial process, i.e. the type of grader, the number of lanes, using downstream packing robots, ...

\section{References}

1. European Commission: Egg Market Situtation (June 1st, 2018). https://circabc. europa.eu/sd/a/18f7766e-e9a9-46a4-bbec-94d4c181183f/17.05.2018_eggs.pdf

2. MOBA. http://www.moba.net/page/en/

3. Feo, T.A., Resende, M.G: Greedy randomized adaptive search procedures. Journal of global optimization 6.2, 109-133 (1995). 